\title{
PULMONARY MICROVESSEL DENSITY IS A MARKER OF ANGIOGENESIS IN CHILDREN AFTER CAVOPULMONARY ANASTOMOSIS
}

Sandra L. Starnes, MD

Brian W. Duncan, MD

James M. Kneebone, MS ${ }^{\mathrm{a}}$

Charles H. Fraga, MS

Shawn States, BS

Geoffrey L. Rosenthal, MD, $\mathrm{PhD}^{\mathrm{b}}$

Flavian M. Lupinetti, MD
Objective: Pulmonary arteriovenous malformations cause progressive cyanosis in children after cavopulmonary anastomosis and may be due to abnormal angiogenesis. We determined the microvessel density, a marker of angiogenesis, in the lungs of children after cavopulmonary anastomosis.

Methods: Lung biopsy specimens were obtained from 8 children after cavopulmonary anastomosis and from 4 control patients. Three of the 8 children undergoing cavopulmonary anastomosis had clinical and angiographic evidence of pulmonary arteriovenous malformations, whereas the other 5 were free of symptoms. Routine histologic and immunohistologic stains were performed with a primary antibody to von Willebrand factor. Microvessel staining for von Willebrand factor was determined for 10 fields (200×) per patient.

Results: Patients with and without pulmonary arteriovenous malformations after cavopulmonary anastomosis demonstrated significantly increased microvessel density compared with control subjects $(32.7 \pm 2.8$ vs $9.3 \pm 4.6$, $P=.02$, and $31.5 \pm 15.7$ vs $9.3 \pm 4.6, P=.01$, respectively). There was no difference in microvessel density in children with and without clinically apparent pulmonary arteriovenous malformations after cavopulmonary anastomosis $(P=.9)$. The children with pulmonary arteriovenous malformations had numerous greatly dilated vessels that were absent in the asymptomatic children after cavopulmonary anastomosis.

Conclusions: After cavopulmonary anastomosis, pulmonary microvessel density is increased even in the absence of clinically apparent pulmonary arteriovenous malformations, supporting the presence of a constant angiogenic stimulus. Children with clinically apparent pulmonary arteriovenous malformations possess large numbers of greatly dilated pulmonary microvessels, which are absent in asymptomatic children after cavopulmonary anastomosis. These results suggest that the transition to clinically apparent pulmonary arteriovenous malformations may be due to mechanisms that lead to vessel dilation and remodeling. (J Thorac Cardiovasc Surg 2000;120:902-8)
From the Divisions of Cardiac Surgery and Cardiology, ${ }^{\mathrm{b}}$ Children's Hospital and Regional Medical Center, Seattle, Wash.

Read at the Eightieth Annual Meeting of The American Association for Thoracic Surgery, Toronto, Ontario, Canada, April 30-May 3, 2000.

Received for publication May 4, 2000; revisions requested June 12, 2000; revisions received June 29, 2000; accepted for publication July 19, 2000.

Address for reprints: Brain W. Duncan, MD, Division of Cardiac Surgery, Children's Hospital and Regional Medical Center, 4800 Sandpoint Way, NE, PO Box 5371/CM-03, Seattle, WA 98105 (E-mail: bdunca@chmc.org).

Copyright (C) 2000 by The American Association for Thoracic Surgery

0022-5223/2000 $\$ 12.00+0 \quad \mathbf{1 2 / 6 / 1 1 0 2 4 8}$

doi: $10.1067 / \mathrm{mtc} .2000 .110248$
Dulmonary arteriovenous malformations (PAVMs) are a cause of progressive cyanosis in children after cavopulmonary anastomosis. The development of PAVMs in these children may be due to the absence of a hepatic inhibitor of angiogenesis in the pulmonary circulation when pulmonary blood flow is derived exclusively from the superior vena cava and is devoid of hepatic venous effluent. ${ }^{1-3}$ Supporting the concept that this is an angiogenically active lesion, we $\mathrm{w}^{4}$ have demonstrated greatly increased numbers of blood vessels that extend far into the periphery of the lung in children with clinically symptomatic PAVMs after cavopulmonary anastomosis. However, if this phenomenon is due to the pres- 
Table I. Demographics of patients after cavopulmonary anastomosis

\begin{tabular}{lcclc}
\hline $\begin{array}{l}\text { Patient No. } \\
\text { (age [y], sex })\end{array}$ & $\begin{array}{c}\text { Clinically } \\
\text { evident PAVMs }\end{array}$ & $\begin{array}{c}\text { Oxygen } \\
\text { saturation (\%) }\end{array}$ & $\begin{array}{c}\text { Time interval since } \\
\text { Glenn shunt }(y)\end{array}$ \\
\hline $1(3.0, \mathrm{~F})$ & Yes & 83 & Heterotaxy & 2.2 \\
$2(12.0, \mathrm{M})$ & Yes & 65 & Heterotaxy & 7.0 \\
$3(7.9, \mathrm{M})$ & Yes & 71 & Transposition, double-inlet left ventricle & 6.6 \\
$4(1.9, \mathrm{M})$ & No & 85 & Tricuspid atresia & 1.3 \\
$5(2.9, \mathrm{M})$ & No & 84 & Pulmonary atresia & 1.7 \\
$6(3.4, \mathrm{~F})$ & No & 84 & Pulmonary atresia & 1.8 \\
$7(3.9, \mathrm{~F})$ & No & 80 & Tricuspid atresia & 3.5 \\
$8(1.8, \mathrm{~F})$ & No & 85 & Hypoplastic left heart syndrome & 1.4 \\
\hline
\end{tabular}

ence of a constant angiogenic stimulus, asymptomatic patients should also demonstrate evidence of increased blood vessel proliferation in the lungs after cavopulmonary anastomosis. Support for this theory comes from contrast echocardiographic studies that have demonstrated increased right-to-left shunting in patients without clinical evidence of PAVMs who have undergone cavopulmonary anastomosis. ${ }^{5,6}$ This shunting can be demonstrated within weeks after cavopulmonary anastomosis and presumably is due to early vascular proliferation that occurs in the lungs of these children.

Microvessel density (MVD) has been used extensively in oncologic studies as a marker of angiogenesis. ${ }^{7-9}$ In the present study, we determined the MVD in the lungs of children after cavopulmonary anastomosis who did not have clinical evidence of PAVMs. We compared this with the MVD in patients with clinically apparent PAVMs after cavopulmonary anastomosis and with the MVD of normal control subjects. Increased MVD in asymptomatic patients after cavopulmonary anastomosis would suggest the presence of a constant angiogenic stimulus in the lungs of these children despite the lack of clinically apparent PAVMs.

\section{Methods}

Patient and control specimens. After obtaining informed consent, we took lung biopsy specimens from 8 patients after cavopulmonary anastomosis at the time of the Fontan procedure. Three of these patients had angiographically documented PAVMs, and the other 5 had no clinical or angiographic evidence of PAVMs. Peripheral lung biopsy specimens were obtained from either the lingula of the left lung or the middle lobe of the right lung with the lung fully inflated with temporary inspiratory hold from the ventilator. Control lung specimens from 4 children with neither congenital heart disease nor significant lung disease were obtained from archived pathology specimens. These control specimens included 2 lung biopsy specimens - the first from a child with an acute viral illness and the second from a child with systemic lupus erythematosus. The other 2 specimens were from autopsiesthe first from a child who died of acute viral myocarditis and the other from a child who died of rabies encephalitis. The study was approved by the institutional review board of Children's Hospital and Regional Medical Center, Seattle, Washington.

Histology and immunohistochemistry. All surgical and autopsy specimens were immediately fixed in formalin and then embedded in paraffin. Sections $5-\mu \mathrm{m}$ thick were cut, deparaffinized in xylene, and rehydrated in graded alcohols. Sections were stained for routine histologic evaluation with hematoxylin and eosin and Movat pentachrome. For immunohistochemical staining, endogenous peroxidase was quenched with $3 \%$ hydrogen peroxide for 10 minutes. Sections were steamed in citrate buffer ( $\mathrm{pH}$ 6.0) for 20 minutes to improve antigen retrieval. After cooling for 20 minutes at room temperature, sections were washed in phosphate-buffered saline solution (PBS, pH 7.4). Rabbit anti-human primary antibody against the endothelial marker, von Willebrand factor (DAKO Corporation, Carpinteria, Calif), diluted $1: 500$ in $1 \%$ bovine serum albumin (BSA) in PBS, was applied to sections. Sections were incubated at room temperature for 60 minutes in a humidity chamber. After being washed in PBS, biotinylated goat anti-rabbit secondary antibody (Vector Laboratories, Burlingame, Calif) diluted 1:500 in 1\% BSA/PBS was applied. Sections were incubated at room temperature for 30 minutes. After the sections were washed in PBS, avidin-biotin enzyme complex was added (Vector Laboratories) and slides were incubated at room temperature for 30 minutes. The sections were developed with diaminobenzidine tetrahydrochloride (Sigma Chemical Co, St Louis, Mo) and counterstained in methyl green. Sections incubated with $1 \%$ BSA in PBS in place of the primary antibody served as negative controls. Microvessels staining positively for von Willebrand factor were counted in 10 high-power fields $(200 \times)$ by 2 investigators. Results for 2 of the 3 patients with clinically apparent PAVMs have been published previously. ${ }^{4}$

Statistical analysis. Statistical analysis was carried out with SAS statistical software, version 6.12 (SAS Institute, Inc, Cary, NC). Analysis of variance was used to compare MVD among patients with and without PAVMs after cavopulmonary anastomosis, as well as control subjects. The Student $t$ test was used to compare ages of patients after cavopulmonary anastomosis and controls. Results are expressed as the mean \pm the standard deviation. 

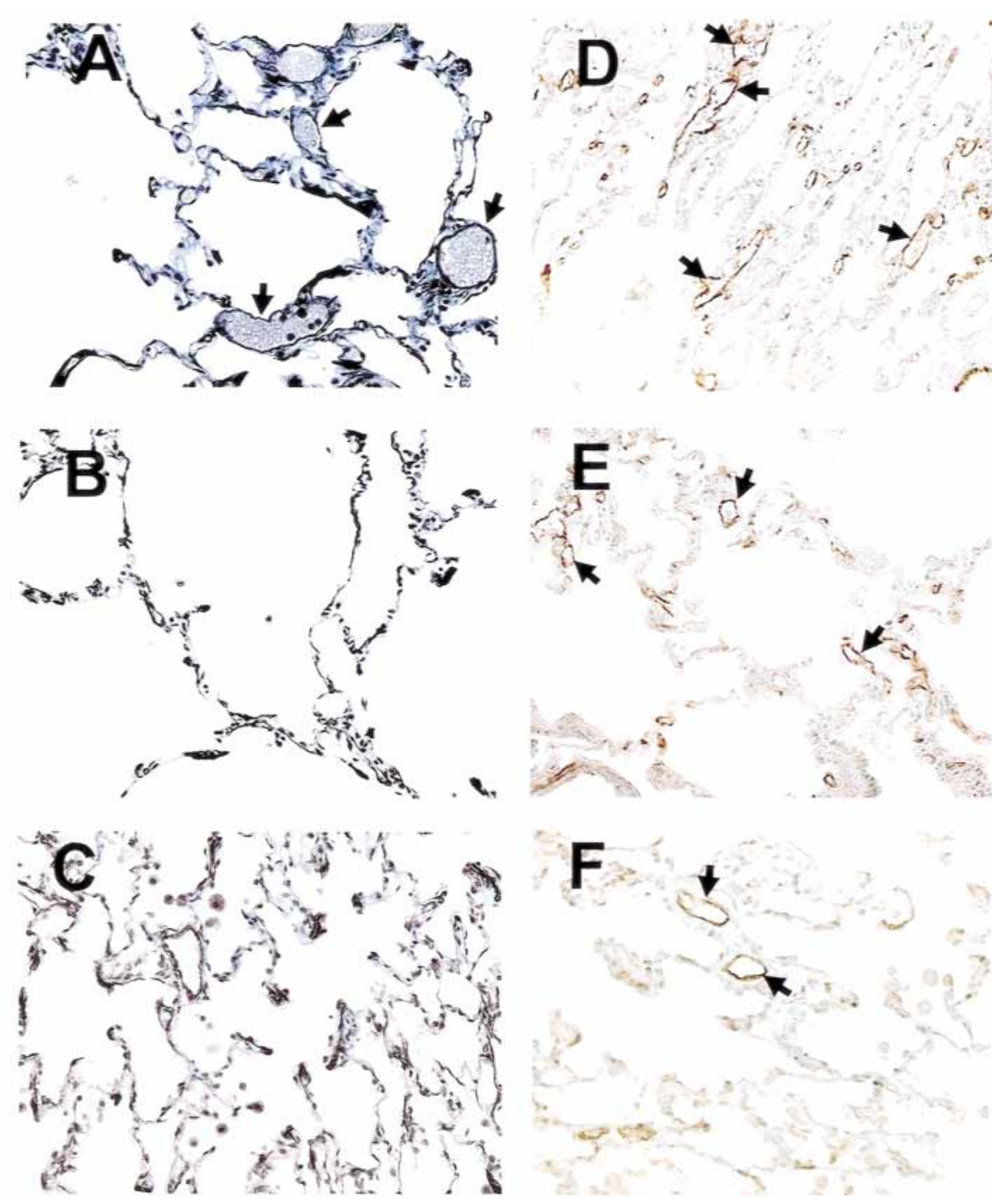

Fig 1. Staining for Movat pentachrome and von Willebrand factor in a patient with a cavopulmonary anastomosis with angiographically documented PAVMs (A and D), a patient without clinical or angiographic evidence of PAVMs after cavopulmonary anastomosis (B and E), and a control patient ( $\mathbf{C}$ and F) (original magnification 200×). Arrows demonstrate vessels staining positively for von Willebrand factor (D, E, and F). Large dilated "lakes" (arrows) are present only in the Movat-stained sections from a patient with symptomatic PAVMs (A).

\section{Results}

Table I shows the demographics of the patients after cavopulmonary anastomosis. There was no statistically significant difference in the ages of patients and controls $(4.6 \pm 3.5$ years vs $5.8 \pm 6.3$ years; $P=.7)$. Fig 1 illustrates staining with Movat pentachrome and von Willebrand factor in 1 patient with symptomatic PAVMs after cavopulmonary anastomosis, in 1 patient without clinically evident PAVMs after cavopulmonary anastomosis, and in 1 control patient. After cavopulmonary anastomosis, both symptomatic and asympto- matic patients had a much higher number of pulmonary microvessels than did control subjects. The control specimens showed no significant abnormalities on microscopic examination; specifically, 1 specimen was completely normal, 2 showed minor focal inflammation, and 1 showed minor focal fibrosis.

After cavopulmonary anastomosis, patients with and without clinical evidence of PAVMs demonstrated significantly increased MVD compared with control subjects $(32.7 \pm 2.8$ vs $9.3 \pm 4.6, P=.02$, and $31.5 \pm 15.7$ vs $9.3 \pm 4.6, P=.01$, respectively). There was no dif- 
ference in MVD in children with and without clinically apparent PAVMs after cavopulmonary anastomosis $(P=.9)$ (Fig 2).

Routine histologic sections stained with hematoxylin and eosin or Movat pentachrome demonstrated a significant qualitative difference between patients who had symptomatic PAVMs after cavopulmonary anastomosis and patients who did not (Fig 1). Sections from the 3 patients with symptomatic PAVMs stained with Movat pentachrome demonstrated numerous greatly dilated vessels, as well as chains of smaller vessels. These chains of numerous small vessels were also present in asymptomatic patients after cavopulmonary anastomosis; however, the large dilated vessels were not present in these patients.

\section{Discussion}

Abnormal angiogenesis is an etiologic factor for numerous medical conditions including tumor metastasis, diabetic retinopathy, rheumatoid arthritis, and gastric ulceration. ${ }^{10}$ PAVMs developing after cavopulmonary anastomosis may also represent a form of abnormal angiogenesis. We undertook the present study to determine whether a sensitive indicator of angiogenesis (MVD) could be used to demonstrate blood vessel proliferation in the lungs of children early after cavopulmonary anastomosis before the development of clinically apparent PAVMs. MVD determined by von Willebrand factor staining has been used extensively as a technique to determine the angiogenic potential of tumors from a wide variety of tissues including breast, colon, and lung. ${ }^{7-9}$

This approach was chosen to provide a histologic correlate to echocardiographic studies that have demonstrated subclinical PAVMs in asymptomatic children after cavopulmonary anastomosis. In these studies, microbubble contrast echocardiography demonstrated right-to-left shunting in children without symptomatic PAVMs as early as 6 weeks after cavopulmonary anastomosis.,6 Our results corroborate these echocardiographic studies, demonstrating increased MVD in patients after cavopulmonary anastomosis. Five of these patients had no angiographic or clinical evidence of PAVMs but clearly had evidence of increased pulmonary vascularity at a microscopic level. In fact, MVD in these asymptomatic patients was similar to that in patients with clinically apparent PAVMs. These increased numbers of small vessels presumably represent the histologic correlate of the right-to-left shunt demonstrated by contrast echocardiography.

$\mathrm{We}^{4}$ have previously described the histologic features of lung tissue from children with clinically apparent

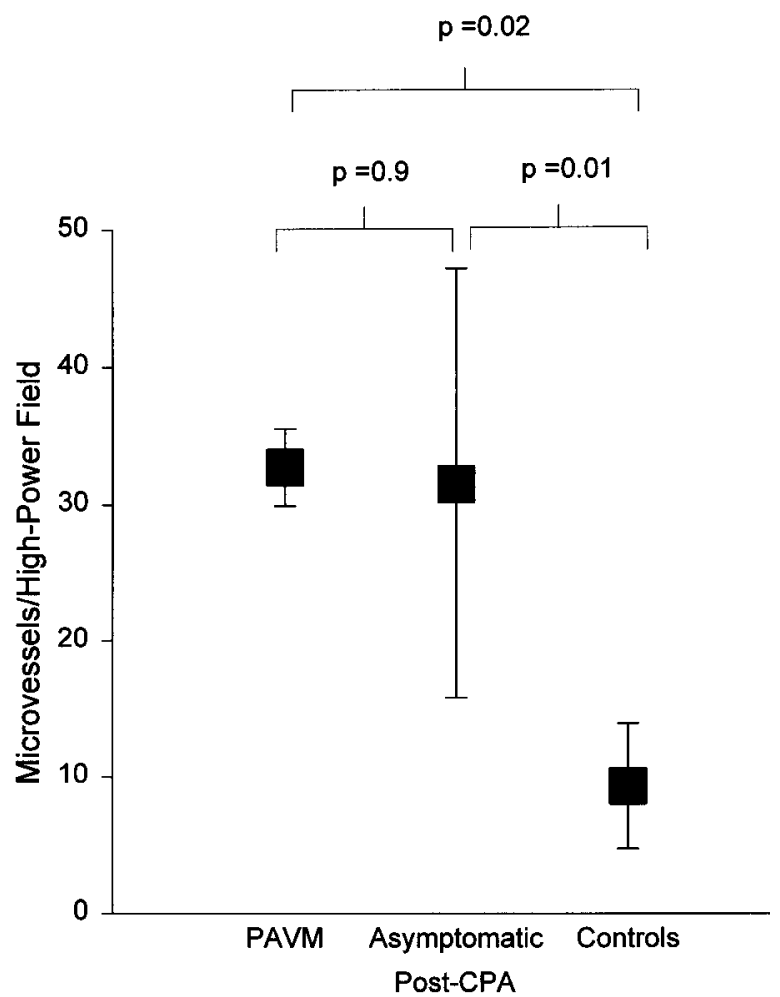

Fig 2. Mean MVD per high-power field $(200 \times)$ in patients after cavopulmonary anastomosis with clinically evident PAVMs, without clinical evidence of PAVMs (Asymptomatic Post-CPA), and controls. Error bars represent standard deviation.

PAVMs after cavopulmonary anastomosis. The predominant histologic feature appeared to be large numbers of blood vessels extending far into the periphery of the pulmonary parenchyma. We described 2 different morphologic types of vessels in these patients-clusters of multiple small blood vessels ("chains") and large, dilated, highly branching vessels ("lakes"). Despite increased MVD in the 5 asymptomatic patients in the present study due to an abundance of vessels with "chains" morphology, none of these patients had the large dilated "lakes" that we found in children with clinically symptomatic PAVMs. The absence of vascular "lakes" in these children probably accounts for their angiographically normal appearance.

The development of dilated, thin-walled vessels appears to represent the histologic transition between the absence of symptoms and clinically apparent PAVMs in children who have had cavopulmonary anastomosis. More important, this finding implies a change in molecular mechanisms that control vascular growth and morphology in this condition. Both groups demon- 
strate evidence of a constant angiogenic stimulus resulting in a quantitative increase in blood vessel number compared with controls. However, patients with clinically apparent PAVMs have a further qualitative difference in vessel morphology from "chains" alone to one in which "lakes" are prevalent.

Potential control mechanisms for this histologic transition would have to account for vascular dilation and wall thinning in addition to vascular proliferation. We speculate that the transition of molecular control for these events involves a change from predominant vascular proliferation in asymptomatic patients after cavopulmonary anastomosis to mechanisms in which vascular remodeling predominates in children with PAVMs. This molecular switch to predominant vascular remodeling represents a fruitful area of future investigation into this condition and a current focus of our laboratory.

The pattern of development of PAVMs in children who have had cavopulmonary anastomosis suggests that the liver may play an important role. PAVMs form in the ipsilateral lung after a classic Glenn shunt ${ }^{11}$ and bilaterally after a bidirectional Glenn shunt or Kawashima procedure. ${ }^{3}$ In each of these instances, PAVMs develop in the pulmonary circulation that is devoid of hepatic venous effluent. Reconstitution of hepatic venous drainage to the lungs results in regression of PAVMs. ${ }^{2,12}$

Further evidence for the role of the liver in this phenomenon comes from the observation that a similar clinical picture is seen in some patients with liver failure who have progressive cyanosis from intrapulmonary shunting. This phenomenon, termed the hepatopulmonary syndrome, closely resembles the angiographic and histologic picture of PAVMs occurring after cavopulmonary anastomosis and resolves after liver transplantation. ${ }^{3,13,14}$ These clinical observations support the hypothesis that PAVMs occurring after cavopulmonary anastomosis may represent new blood vessel growth due to lack of a hepatic inhibitor of angiogenesis. $\mathrm{We}^{1}$ have partially purified a factor from the conditioned media of cultured hepatocytes that inhibits endothelial cell proliferation. The present study does not directly address the role of the liver in the development of PAVMs after cavopulmonary anastomosis. Absence of a liver factor may be the cause of this presumed vascular proliferation that all children demonstrate after cavopulmonary anastomosis, as well as influencing the vascular remodeling that leads to clinically apparent PAVMs.

On the basis of our previous work suggesting that these lesions are due to abnormal angiogenesis under hepatic control, we have interpreted that vascular pro- liferation plays a role in the development of these lesions, at least early in the course of their development. However, an alternative theory is that these abnormal blood vessels seen in children after cavopulmonary anastomosis are actually dilated collaterals that form from embryonic vascular channels already present in the lung. ${ }^{15} \mathrm{We}^{4}$ have previously shown that clinically apparent PAVMs are negative for proliferating cell nuclear antigen, a marker of vascular proliferation. This result suggests that at the latest stage of PAVM development, these lesions are no longer highly proliferative, which further underlines the importance of vascular remodeling and dilatation in the etiology of these lesions. We are currently studying lung tissue from children with early (asymptomatic) and late (clinically apparent) PAVMs using protein and mRNA techniques for markers of angiogenesis and vascular remodeling. Ideally, these studies will further define the exact contribution of these processes to the development of these lesions.

A limitation of the present study is the small number of patients examined. However, increased MVD was a constant finding in all of the patients who were examined after cavopulmonary anastomosis. MVD in all of these patients exceeded that in any of the control subjects. In addition, the fact that these biopsy specimens were obtained from peripheral pulmonary parenchyma confirms the diffuse nature of this process, which may be even more advanced centrally in the lung. Another limitation of the study is the lack of completely normal controls; however, all control patients had an acute onset of illness of less than 1 week in duration before biopsy or autopsy and demonstrated essentially normal pulmonary architecture. A further limitation exists in failing to control for hypoxia, which itself is a potent stimulator of angiogenesis and which was present in all of the patients after cavopulmonary anastomosis. Further studies determining MVD in lung tissue from hypoxic children who have not undergone cavopulmonary anastomosis or animal models might help to address this concern.

\section{Summary}

The development of PAVMs may represent an abnormal form of angiogenesis under hepatic control, which has led us to hypothesize that asymptomatic patients should have subclinical manifestations of angiogenesis after cavopulmonary anastomosis. Our results suggest that a constant angiogenic stimulus is present after cavopulmonary anastomosis, with asymptomatic patients demonstrating 3-fold higher MVD than control subjects. A comparison of the histologic features 
between asymptomatic patients and children with PAVMs after cavopulmonary anastomosis demonstrates a histologic transition to the development of dilated thin-walled vessels extending into the lung periphery. Identifying mechanisms that control vascular remodeling in this lesion, as well as those that control vascular proliferation, will be important to the ultimate understanding of its etiology.

\section{REFERENCES}

1. Marshall B, Duncan B, Jonas R. The role of angiogenesis in the development of pulmonary arteriovenous malformations in children after cavopulmonary anastomosis. Cardiol Young 1997;7:370-4.

2. Knight W, Mee R. A cure for pulmonary arteriovenous fistulas? Ann Thorac Surg 1995;59:999-1001.

3. Srivastava D, Preminger T, Lock J, Mandell V, Keane J, Mayer J, et al. Hepatic venous blood and the development of pulmonary arteriovenous malformations in congenital heart disease. Circulation 1995;92:1217-22.

4. Duncan B, Kneebone J, Chi E, Hraska V, Isik F, Rosenthal G, et al. A detailed histologic analysis of pulmonary arteriovenous malformations in children with cyanotic congenital heart disease. J Thorac Cardiovasc Surg 1999;117:931-8.

5. Bernstein H, Brook M, Silverman N, Bristow J. Development of pulmonary arteriovenous fistulae in children after cavopulmonary shunt. Circulation 1995;92(Suppl):II-309-14.

6. Chang R, Atkinson D, Jensen R, Drant S, Laks H, Alejos J. Bubble contrast echocardiography in detecting pulmonary arteriovenous malformation in children with cyanotic congenital heart disease. Circulation 1998;98(Suppl):I-618.

7. Weidner N, Semple J, Welch W, Folkman J. Tumor angiogenesis and metastasis - correlation in invasive breast carcinoma. N Engl J Med 1991;324:1-8.

8. Takahashi Y, Kitadai Y, Bucana C, Cleary K, Ellis L. Expression of vascular endothelial growth factor and its receptor, KDR, correlates with vascularity, metastasis, and proliferation of human colon cancer. Cancer Res 1995;55:3964-8.

9. Yamazaki K, Shosaku A, Takekawa H, Sukoh N, Watanabe N, Shigeaki O, et al. Tumor angiogenesis in human lung adenocarcinoma. Cancer 1994;74:2245-50.

10. Folkman J. Angiogenesis in cancer, vascular, rheumatoid and other disease. Nature Med 1995;1:27-31.

11. McFaul R, Tajik A, Mair D, Danielson G, Seward J. Development of pulmonary arteriovenous shunt after superior vena cava-right pulmonary artery (Glenn) anastomosis. Circulation 1977;55:212-6.

12. Shah M, Rychik J, Fogel M, Murphy J, Jacobs M. Pulmonary AV malformations after superior cavopulmonary connection: resolution after inclusion of hepatic veins in the pulmonary circulation. Ann Thorac Surg 1997;63:960-3.

13. Lange P, Stoller J. The hepatopulmonary syndrome. Ann Intern Med 1995;122:521-9.

14. Stoller J, Moodie D, Schiavone W, Vogt D, Broughan T, Winkelman E, et al. Reduction of intrapulmonary shunt and resolution of digital clubbing associated with primary biliary cirrhosis after liver transplantation. Hepatology 1990;11:54-8.

15. Pandurangi UM, Shah MJ, Murali R, Cherian KM. Rapid onset of pulmonary arteriovenous malformations after cavopulmonary anastomosis. Ann Thorac Surg 1999;68:237-9.

\section{Discussion}

Dr Richard A. Jonas (Boston, Mass). When Dr Duncan was with us at Children's Hospital in Boston, he and I had the exciting opportunity to collaborate with the godfather of angiogenesis, Dr Judah Folkman. Working in conjunction with one of the surgical residents in Dr Folkman's laboratory, Dr Blair Marshall, we were well on the way to purifying a hepatic-derived angiogenic inhibitory factor in an animal model. I am delighted to see that Dr Duncan has continued his interest in this area in Seattle and is presently undertaking both laboratory and clinical studies, as Dr Starnes has described.

The main finding of this report is a very important one, namely, that it appears that any child who undergoes cavopulmonary anastomosis is susceptible to a process of vascular proliferation and must ultimately be at risk for the development of symptomatic PAVMs resulting in important cyanosis. Thus, it would seem likely that a philosophy of eventually converting all patients who have had a bidirectional Glenn shunt to a Fontan-type circulation is justified.

Can you speculate on why some patients seem to be predisposed to the rapid development of symptomatic PAVMs? For example, the patient with heterotaxy after a Kawashimatype procedure appears particularly susceptible to accelerated development of clinically important PAVMs. What is it about this population or other individual patients with accelerated development of PAVMs that distinguishes them from patients who have only slow progression of cyanosis?

Second, how are you presently studying this problem in a laboratory model? In Boston we were using a cell culture model with bovine endothelial cells stimulated by a hepatic factor derived from rat liver. Do you have a large animal model and can you share any preliminary information with us regarding the link between a hepatic-derived factor and proliferation of pulmonary blood vessels?

Dr Starnes. Thank you, Dr Jonas, for those comments.

The development of PAVMs is certainly a complicated process, and we believe that a liver factor is probably the major factor; however, undoubtedly multiple other factors are involved. One example is cyanosis itself. Hypoxia is a known stimulus for angiogenesis. In fact, we recently reported that patients with cyanotic congenital heart disease have elevated levels of vascular endothelial growth factor, a very potent angiogenic stimulus, in their serum. Thus, the degree of cyanosis may be one factor that is more important in some children.

In patients with heterotaxy, the difference in pressure between the caval system and the splanchnic venous system may be an additional factor that is important in the rapid progression of PAVMs.

Regarding the second question, we agree that an animal model is of the utmost importance in determining the mechanisms involved in this process. First, an animal model would allow us to have essentially limitless amounts of tissue to study. Another reason is that we could study the progression of these lesions over a long period. However, most important, the use of an animal model would allow us to develop some medical therapies for this problem. A growing number of antiangiogenic 
agents are available, such as interferon- $\alpha$ or inhibitors of vascular endothelial growth factor, that we could use in an animal model to see whether we can induce these lesions to regress.

For these reasons, we have developed a small animal model of PAVMs after cavopulmonary anastomosis in a rat. This model successfully reproduces the condition seen in human beings. Angiographic evidence of PAVMs occurs 12 months after cavopulmonary anastomosis in this model, whereas at earlier time points the angiograms were normal. However, using the same methods described in this report, we found evidence of vascular proliferation in this animal model as early as 2 months after surgery.

We chose a small animal model instead of a large animal model for a couple of reasons. First is the expense. We can study a larger number of rats than we can larger animals. The second reason is the availability of agents for the molecular analysis. These agents are widely available in a rat model.

Regarding the third question, the identification of this hepatic factor was started at Boston Children's with Dr Folkman, and we are very fortunate to have that as our background. Over the past couple of years in Seattle, we have focused on our animal model and these studies in human tissue, but in the near future we will be focusing on the hepaticderived factor again to see whether we can further purify and finally identify this factor. The recent development of immortalized hepatic cell lines would make this much easier to do today than it was even a few years ago. 